\title{
Multifractal Analysis of the Dynamical Heterogeneity in a Two-Dimensional Supercooled Liquid
}

\author{
W. Sakikawa and O. Narikiyo \\ Department of Physics, Kyushu University, Fukuoka 810-8560, Japan
}

(May, 2002)

\begin{abstract}
The dynamical heterogeneity in supercooled liquids measured by a molecular dynamics simulation has been quantified on the basis of the multifractal formalism. The singularity spectrum becomes broader as the glass transition is approached. This behavior is similar to that observed in the case of the Anderson-localization transition.
\end{abstract}

Keywords: supercooled liquid, glass, dynamical heterogeneity, multifractal

The dynamical heterogeneity in supercooled liquids has been studied intensively in recent years. It can be quantified by introducing the concept of bond-breakage and the distribution of the broken bonds near the glass-transition is found to be similar to that of the spin state of the Ising model near the critical point. [2] The bond-breakage corresponds to the collective jump motion of particles among cages.

On the other hand, we have analyzed the dynamical heterogeneity of the critical spin state of the Ising model on the basis of the multifractal formalism. 33 In this Short Note we try to combine above two approaches and quantify the dynamical heterogeneity observed in a molecular dynamics simulation of a supercooled liquid in terms of multifractality. The so-called cooperatively rearranging regions (CRR) can be identified with weakly bonded regions with various sizes which form multifractal structure.

We use the same model as in Ref. 1, the binary mixture of soft core particles. Two species of particles, 1 and 2 , with the size $\sigma_{1}$ and $\sigma_{2}$, and the mass $m_{1}$ and $m_{2}$ are considered. The interaction potential is given by

$$
v_{\alpha \beta}(r)=\epsilon \cdot\left(\frac{\sigma_{\alpha \beta}}{r}\right)^{12}
$$

where $r$ is the distance between two particles and $\sigma_{\alpha \beta}=\left(\sigma_{\alpha}+\sigma_{\beta}\right) / 2$ with $\alpha, \beta=1,2$.

The molecular dynamics simulation is done using the second order symplectic integrator [4] and the microcanonical ensemble. The temperatures in the following are determined by the kinetic energy.

The bond is introduced as in Ref. 1 when

$$
r_{i j}\left(t_{0}\right) \leq 1.1 \sigma_{\alpha \beta},
$$

where $r_{i j}\left(t_{0}\right)$ is the distance between $i$-th particle of species $\alpha$ and $j$-th particle of species $\beta$ at time $t_{0}$. The position of the bond is represented by the midpoint of the two particles at this time. The bonds are unambiguously defined [2] owing to the sharpness of the first peak of their pair correlation function. The bond is regarded as surviving at time $t_{0}+t$ when

$$
r_{i j}\left(t_{0}+t\right) \leq 1.6 \sigma_{\alpha \beta} .
$$

The number of surviving bonds $N_{\text {suv }}\left(t_{0}+t\right)$ is fitted by

$$
N_{\text {suv }}\left(t_{0}+t\right)=N_{\text {suv }}\left(t_{0}\right) \exp \left(-t / \tau_{\mathrm{b}}\right),
$$

introducing the life-time of bonds $\tau_{\mathrm{b}}$. In the following the broken bonds are determined for $t=0.05 \tau_{\mathrm{b}}$.

In our simulation the followings are fixed. Two species of particles have the same particle number, $N_{1}=N_{2}=80000$. The size and mass ratios are chosen as $\sigma_{2} / \sigma_{1}=1.4$ and $m_{2} / m_{1}=2$ respectively. The time step of the integration $\Delta t$ is chosen as $\Delta t=0.005 \tau_{1}$ with $\tau_{1}=\left(m_{1} \sigma_{1}^{2} / \epsilon\right)^{1 / 2}$. The potential is truncated at $r=4.5 \sigma_{1}$. The particle density is chosen as $n=0.8 / \sigma_{1}^{2}$. The simulations were performed on our personal computer with Pentium- 4 CPU.

In Fig. 1 the snapshot of the broken bonds is shown where the position of the bond is defined at time $t_{0}$ as mentioned in the above. In Figs. 1(a) and 1(b) the temperatures are chosen as $T=2.54$ and $T=0.859$ respectively in the unit of $\epsilon / k_{\mathrm{B}}$. These two states are realized starting from an initial state with the temperature 9.69 and cooled in a stepwise manner as in Ref. 1. In the stepwise cooling process the temperatures in the first, second, third and fourth stages are $9.69,2.54,1.43$ and 0.859 respectively. For the former state the temperature was changed from 9.69 to 2.54 after spending 5000 $\Delta t$ at the first stage and the broken bonds are observed at the second stage with $t_{0}=5000 \Delta t$. For the latter state the temperature was changed successively from $9.69,2.54,1.43$ to 0.859 after spending $5000 \Delta t$ at the first, second and third stages each and the broken bonds are observed at the fourth stage with $t_{0}=60000 \Delta t$. The times 
$t_{0}$ and $t$ are measured from the instance of the temperature change to the stage of the observation. The life-times of bonds were measured to be $\tau_{\mathrm{b}}=4.09 \times 10^{3} \Delta t$ for $T=2.54$ and $\tau_{\mathrm{b}}=2.43 \times 10^{5} \Delta t$ for $T=0.859$.

Qualitatively the snapshot shows a multifractal behavior and the degree of intermittency increases as the temperature is decreased. In the following we quantify this observation on the basis of the multifractal formalism.

The multifractality is quantified by the singularity spectrum, $f(\alpha)$, and the spectrum is obtained by the following formulae, [5]

$$
\alpha(q)=\sum_{k=1}^{N_{L}} \mu_{k}(q, l) \ln \mu_{k}(1, l) / \ln (l / L)
$$

and

$$
f(\alpha(q))=\sum_{k=1}^{N_{L}} \mu_{k}(q, l) \ln \mu_{k}(q, l) / \ln (l / L)
$$

with

$$
\mu_{k}(q, l)=\left\{\mu_{k}(l)\right\}^{q} / \sum_{k^{\prime}=1}^{N_{L}}\left\{\mu_{k^{\prime}}(l)\right\}^{q} .
$$

Here the measure, $\mu_{k}(l)$, is defined as the probability to find a broken bond in the $k$-th square box of the linear dimension $l$ in a snapshot of broken bonds. The total number of the boxes $N_{L}=(L / l) \times(L / l)$ where $L$ is the linear dimension of the simulation region with periodic boundary condition.

In Fig. 2 the singurarity spectra for $T=2.54$ and $T=0.859$ are shown. The dots represent the data set $(\alpha(q), f(\alpha(q)))$ determined by the above formulae and should be tangential to $f(\alpha)=2$ and $f(\alpha)=\alpha$. The maximum $\alpha_{\max }$ and minimum $\alpha_{\min }$ are determined by the intercept of the spectrum extrapolated to the $\alpha$-axis and correspond to the singularity exponent for the box with the lowest density of broken bonds and the one with the highest density respectively.

From the data in Fig. 2 we can quantify the degree of intermittency. The singularity spectrum becomes broader as the temperature is decreased approaching the glass transition. At the same time $\alpha_{\max }$ becomes larger and $\alpha_{\min }$ becomes smaller. Namely $\alpha_{\max }=2.74$ and $\alpha_{\min }=1.66$ for $T=2.54$ and $\alpha_{\max }=2.97$ and $\alpha_{\min }=1.57$ for $T=0.859$. This finding is similar to that reported in the case of the Anderson localization [5] where the singularity spectrum becomes broader as the strength of the impurity potential is increased and the localization transition is approached. In both cases the degree of intermittency corresponds to that of localization.

In conclusion we have quantified the dynamical heterogeneity in a model supercooled liquid measured by a molecular dynamics simulation on the basis of the multifractal formalism. In supercooled liquids various sizes of CRR are formed and constitute multifractal structures. The degree of intermittency increases approaching the glass transition. Since our simulation parameters are still far from those for the glass transition, we need further study to elucidate the nature of the glass transition.

The authors are grateful to Jun Matsui for enlightening discussions on the molecular dynamics simulation. This work was supported in part by a Grand-in-Aid for Scientific Research from the Ministry of Education, Culture, Sports, Science and Technology of Japan.

[1] This paper has been submitted to the Short Note section of J. Phys. Soc. Jpn.

[2] R. Yamamoto and A. Onuki: J. Phys. Soc. Jpn. 66 (1997) 2545.

[3] W. Sakikawa and O. Narikiyo: J. Phys. Soc. Jpn. 71 (2002) 1200.

[4] M. Tuckerman, B. J. Berne and G. J. Martyna: J. Chem. Phys. 97 (1992) 1990.

[5] M. Schreiber: Computational Physics, ed. K. H Hoffmann and M. Schreiber (Springer-Verlag, Berlin, 1996$)$ p.147.

FIG. 1. The snapshot of the broken bonds for (a) $T=2.54$ and (b) $T=0.859$.

FIG. 2. The singularity spectrum for $T=2.54$ and $T=0.859$ with $L / l=30$. 


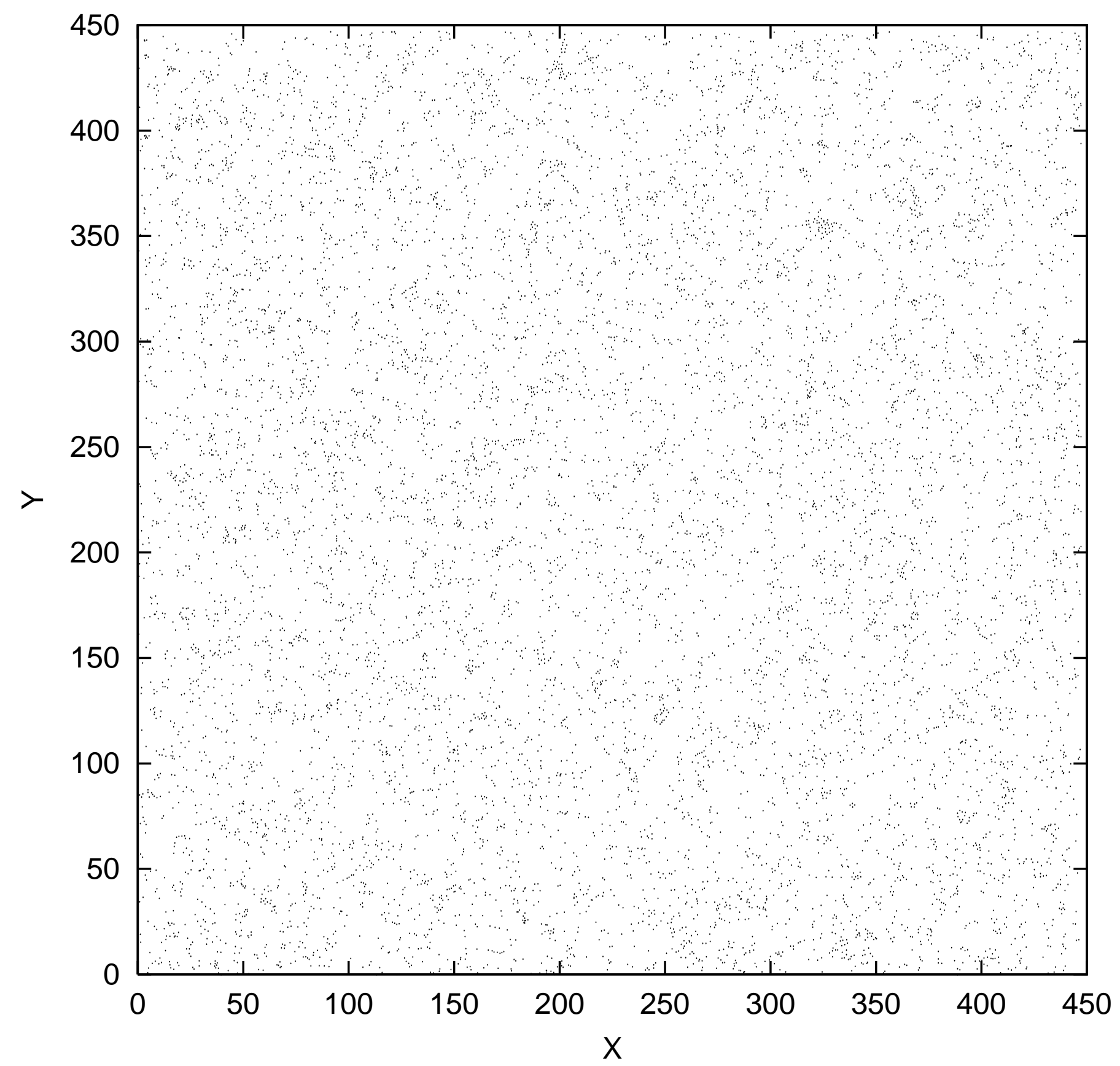




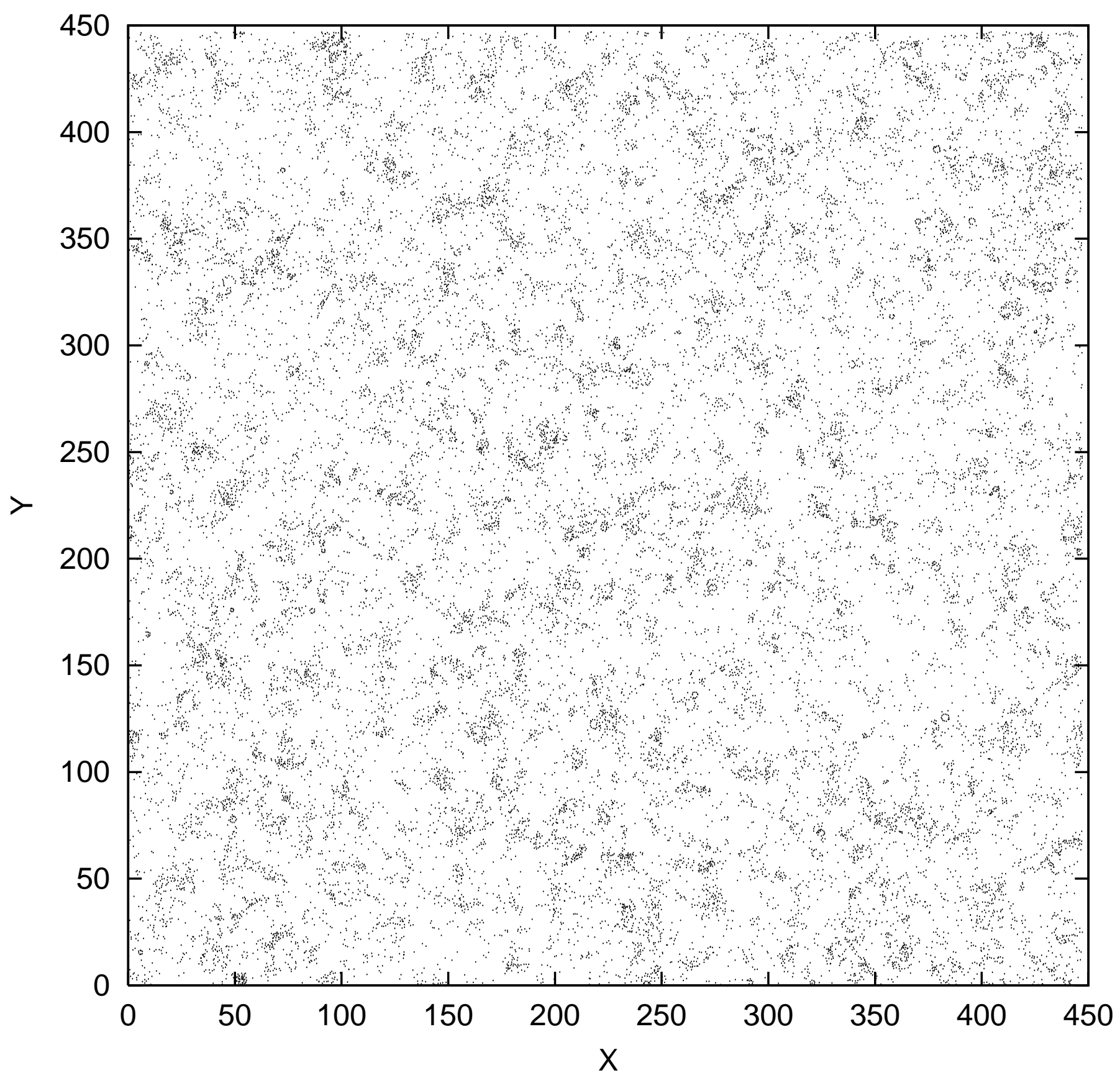




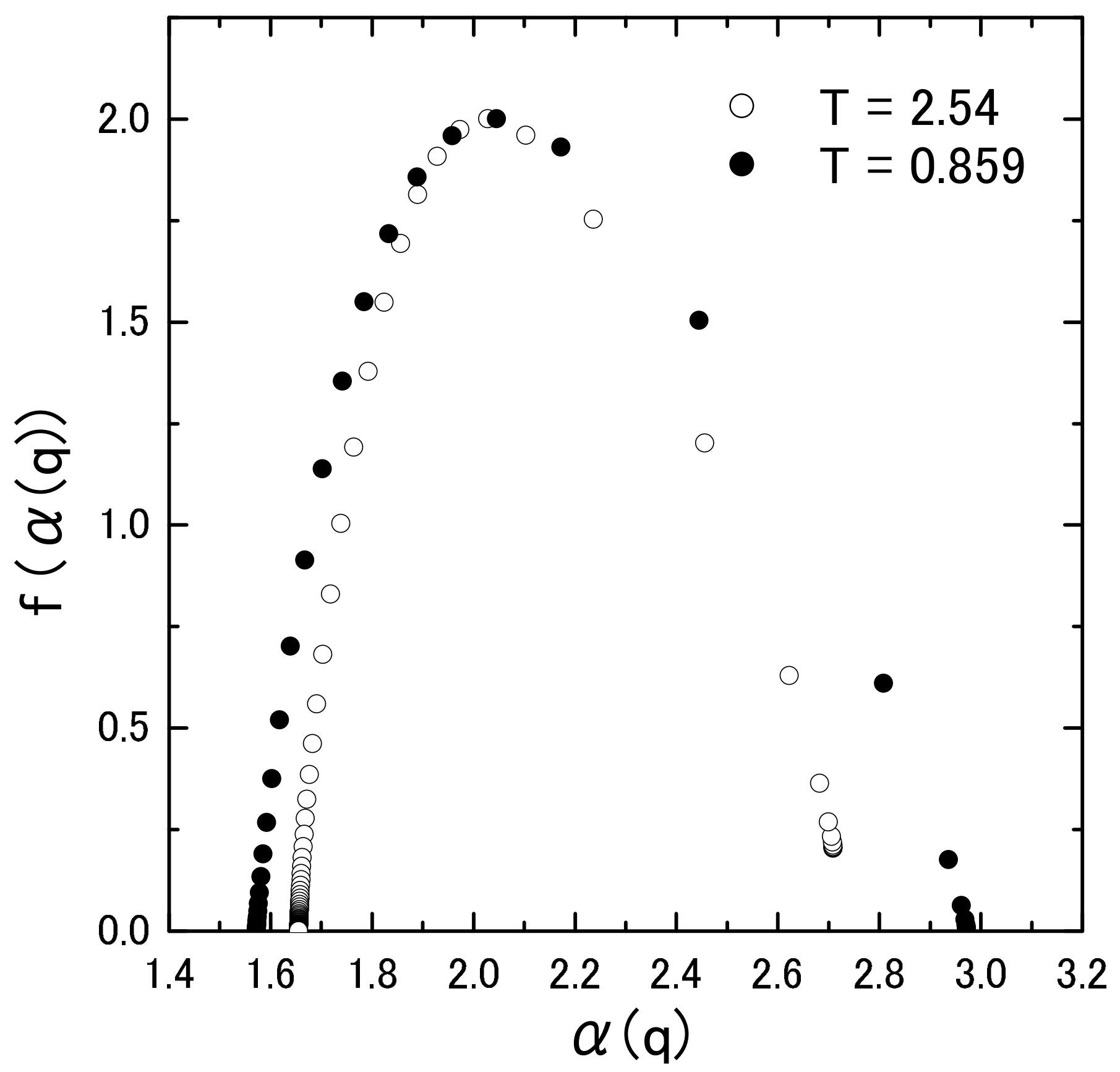

